\title{
REVUE
}

\section{L'EMPLOI DE L'ÉNERGIE ÉLECTRIQUE DANS LES INSTALLATIONS MODERNES DE LAITERIE}

\author{
par \\ G. GENIN \\ Ingénieur chimiste E. P. C.
}

L'emploi de l'énergie électrique dans les laiteries modernes présente des avantages considérables, car il permet d'assurer dans les usines une très grande propreté, facilite considérablement le contrôle des diverses opérations et supprime un grand nombre d'odeurs dues à l'utilisation de différents combustibles. L'emploi du courant électrique dans les laiteries constitue également pour les producteurs d'électricité un débouché très intéressant, d'autant plus que la consommation reste sensiblement constante pendant les 24 heures de la journée.

Deux revues anglaises « The Electrical Times " et " The Electrical Review " ont publié il y a quelques mois des renseignements précieux sur l'électrification des laiteries, en indiquant dans quels domaines l'emploi du courant électrique présente le maximum d'avantages et une de ces revues a donné une description rapide des installations réalisées dans une grande laiterie de Londres : les "London Wholesale Dairies " qui alimentent en lait la plus grande partie de la ville de Londres.

Nous indiquerons tout d'abord de quelle façon le courant électrique peut être utilisé pour les diverses opérations que l'on effectue dans une laiterie.

\section{Homogénéisation.}

Le lait est en général livré aux laiteries, soit en bidons, soit en wagons-réservoirs. Il doit être généralement stocké dans de grandes cuves, après qu'il a été pesé et c'est de ces cuves qu'il est dirigé par pompage vers les différentes salles de traitement. Le lait destiné à être consommé directement est généralement homogénéisé; ce traitement qui se fait sur le lait préalablement réfrigéré a pour objet de réduire les dimensions des globules de matière grasse et de les répartir uniformément dans le liquide. Ce traitement s'effectus dans des homogénéiseurs, le plus souvent à commande électrique, et par exemple un appareil capable de traiter 1.200 litres de lait à l'heure, exige pour sa commande un moteur de $15 \mathrm{CV}$.

\section{Lavage des réci pients à lait.}

Le lavage des bidons, boîtes et bouteilles destinés à contenir le 
lait, s'effectue aujourd'hui dans une usine moderne d'une façon entièrement automatique. La commande des appareils nécessite une dépenseimportante d'énergie électrique. Par exemple, les récipients qui se déplacent dans la machine à laver et à rincer à une cadence de 3 à 10.000 bouteilles à l'heure, sont placés sur des convoyeurs qui les font passer dans les différentes parties de la machine à laver. La durée du passage d'une bouteille dans une machine de ce genre est en moyenne de 10 minutes et pendant ce temps, la bouteille est lavée plusieurs fois par des jets de lessive alcaline, elle est ensuite rincée à l'eau chaude, puis à l'eau froide et finalement séchée par un courant d'air chaud. Une machine à laver susceptible de traiter 7.000 bouteilles à l'heure comprend plusieurs moteurs électriques pour assurer son fonctionnement, par exemple un moteur de $13 \mathrm{CV}$ pour la commande du convoyeur, un moteur de $12 \mathrm{CV}$ pour la pompe à lessive et un de $8 \mathrm{CV}$ pour la pompe à eau froide.

\section{3o Remplissage.}

Le remplissage est également une opération qui est presque toujours automatique. Les bouteilles sont à cet effet placées sur un disque mobile et, à un certain moment, elles sont poussées vers le haut sur une valve de remplissage, de telle sorte que sous l'action de la poussée de la bouteille, une quantité parfaitement déterminée de liquide s'écoule dans le récipient. A ce moment, la bouteille redescend vers le bas, l'ouverture du passage du liquide se ferme et le récipient est dirigé vers la machine à capsuler qui fonctionne d'ailleurs d'une façon analogue. En général, il suffit d'un moteur de $1 \mathrm{CV}$ pour commander à la fois la machine de remplissage et la machine à capsuler qui souvent d'ailleurs ne constituent qu'un seul appareil.

\section{Pasteurisation.}

La pasteurisation s'effectue le plus souvent après mise en bouteille du lait. On utilise à cet effet de grands récipients métalliques qui contiennent des paniers à claire-voie portant chacun 20 à 30 bouteilles. Ces paniers se déplacent lentement dans le pasteurisateur, de telle sorte que la durée de séjour du lait dans l'appareil est de 2 heures. Les paniers sont ensuite dirigés vers un réfrigérant alimenté par une saumure. Le fonctionnement de ces appareils, ainsi que le chauffage des pasteurisateurs, peuvent être assurés par le courant électrique.

On peut utiliser dans le même ordre d'idées des chaudières électriques destinées à produire la vapeur et l'eau chaude nécessaíres aux besoins de l'usine.

\section{$5^{\circ}$ Fabrications diverses.}

Le lait est une matière première à la base d'un grand nombre de 
fabrications, et pour ne citer que deux exemples nous indiquerons la fabrication du lait en poudre et celle des produits alimentaires pour bestiaux et volailles.

La fabrication du lait en poudre s'effectue soit par atomisation, soit par séchage sur tambour. Le chauffage des appareils peut parfaitement s'effectuer au moyen du courant électrique qui produira la vapeur destinée à traverser les cylindres chauffants ou qui réchauffera l'air envoyé dans la chambre d'atomisation. De même, le courant électrique assure la marche de ces appareils et de tous leurs accessoires.

Dans la fabrication du beurre, la séparation préalable de la crème s'effectue dans des écrémeuses commandées par des moteurs électriques et une écrémeuse d'une capacité de 450 litres est munie généralement d'un moteur d'un demi cheval-vapeur.

Voici d'ailleurs la description rapide d'une installation anglaise où on a fait appel, à tous les stades de la production, au courant électrique.

Le lait livré aux London Wholesale Dairies arrive à l'usine par wagon-réservoir et il est transféré au moyen de l'air comprimé dans les réservoirs installés en sous-sol. Chaeun de ces réservoirs est muni d'un agitateur commandé par un moteur de $1 \mathrm{CV}$ et le lait qu'il contient est envoyé par pompage vers les diverses salles de traitement comprenant également des réservoirs munis d'agitateurs. On trouve dans ces salles les pompes à lait commandées par des moteurs électriques de 5 à $10 \mathrm{CV}$, des pompes à eau chaude, des réfrigérants, des écrémeuses, des homogénéiseurs, etc.

Les cuves de pasteurisation sont également munies de dispositifs fonctionnant par le courant électrique et par exemple, chaque cuve est éclairée intérieurement pour vérifier la marche de l'opération par l'intermédiaire de hublots; tous les robinets placés sur ces cuves et sur les appareils accessoires sont contrôlés automatiquement à partir d'un tableau central sur lequel on a installé des enregistreurs de température, des auto-régulateurs de pression, qui commandent la circulation du lait chaud, du lait froid et de l'eau.

L'installation de stérilisation est divisée en trois parties dans lesquelles on effectue successivement le lavage et le remplissage des récipients, leur stérilisation et leur empaquetage. D'autres ateliers sont également prévus pour le remplissage de bidons à lait, et les manutentions dans tous ces ateliers sont commandées électriquement.

Le stockage du lait s'effectue dans des chambres froides. L'installation frigorifique comprend trois groupes motocompresseurs de $100 \mathrm{CV}$, refroidis par deux pompes de circulation de $10 \mathrm{CV}$, tandis 
que la circulation de la saumure est assurée par deux pompes de $20 \mathrm{CV}$ et deux pompes de $15 \mathrm{CV}$.

On a également fait appel au courant électrique dans la chaufferie. On utilise en effet des chaudières à foyer automatique nécessitant un moteur de $0,75 \mathrm{CV}$ par grille, un moteur de $5 \mathrm{CV}$ par ventilateur de tirage, et un moteur de $20 \mathrm{CV}$ pour la projection du charbon pulvérisé servant de combustible.

Nous terminerons enfin en rappelant le rôle que joue le courant électrique dans la commande des installations de signalisation, du contrôle thermostatique de circulation d'eau et de saumure, du réseau téléphonique et des horloges électriques synchrones. La puissance consommée par l'usine atteint $750 \mathrm{kw}$.

\section{BIBLIOGRAPHIE ANALYTIQUE}

\section{LES LIVRES}

Bonanos (C.). - Le buffle en Grèce. Thèse Doct. Vét., Paris, 1936. Br., 69 p. Jouve et Cie, éditeurs, 15, rue Racine.

Si les buffles ont la plus grande importance économique en Asie où ils forment un troupeau de près de 50 millions de têtes, ils sont aussi exploités dans quelques pays de l'Europe centrale et méridionale, notamment en Grèce, où, pour les départements de Macédoine et de Thrace, on compte environ 50.000 sujets.

Les animaux sont entretenus pendant presque toute l'année sur les prairies communales, souvent desséchées pendant l'été, et dont la végétation est constituée de plantes pəu nutritives ; ils consomment aussi très volontiers les plantes des marécages. Le supplément de nourriture est constitué par de la paille de blé, des feuilles et des tiges de maïs, puis, dans des cas très rares, par un peu d'orge ou d'avoine; enfin, à la ration des bufflesses laitières, on ajoute quelquefois des aliments concentrés, d'origine industrielle. L'habitation est très précaire.

Le buffle est utilisé comme producteur de travail, et à ce titre il est, dans ces pays, incomparable ; comme producteur de viande, lorsqu'il a atteint un âge avancé, 15 à 25 ans. La production du lait est aussi fort importante, et B. C. se demande si, en Grèce où le lait est peu abondant, la bufflesse laitière pourrait être entretenue en stabulation, et exploitée selon le mode intensif. Cette question est toute d'actualité en raison des travaux de drainage entrepris depuis 1929, en Macédoine ; il semble qu'on puisse y répondre par l'affirmative, vu les exemples donnés en beaucoup d'autres pays.

D'après les renseignements fournis par les paysans, la bufflesse grecque ne donnerait guère que 500 à 800 litres de lait par an, y compris le lait consommé par le bufflon. Les statistiques officielles de Grèce sur la production et la consommation de lait feraient ressortir le rendement moyen à 887 litres, auxquels s'ajouterait le lait prélevé par le jeune, ce qui constitue un total très 